\title{
Evaluation of Cardinal Direction Developments between Moving Points
}

\author{
Tao Chen, Hechen Liu \& Markus Schneider* \\ Department of Computer \& Information Science \& Engineering \\ University of Florida \\ Gainesville, FL 32611, USA \\ \{tachen, heliu, mschneid\}@cise.ufl.edu
}

\begin{abstract}
Recently, a wide range of applications like hurricane research, fire management, navigation systems, and transportation has shown increasing interest in managing and analyzing space and time-referenced objects, so-called moving objects, that continuously change their positions over time. In the same way as moving objects can change their location over time, the spatial relationships between them can change over time. An important class of spatial relationships are cardinal directions like north and southeast. In spatial databases and GIS, they characterize the relative directional position between static objects in space and are frequently used as selection and join criteria in spatial queries. Transferred to a spatiotemporal context, the simultaneous location change of different moving objects can imply a temporal evolution of their directional relationships, called development. The goal of this paper is to illustrate, explain, and formally define cardinal direction developments between two moving points.
\end{abstract}

Categories and Subject Descriptors: H.2.8 [Database Management]: Spatial databases and GIS

General Terms: Design, Languages

Keywords: Moving object, cardinal direction, cardinal direction development, spatio-temporal directional predicate

\section{INTRODUCTION}

Objects that continuously change their positions over time, so-called moving objects, have recently received a lot of interest. Examples are moving points like vehicles, mobile devices, and animals, for which the time-dependent position is relevant. Temporal movements of spatial objects induce

\footnotetext{
*This work was partially supported by the National Science Foundation under grant number NSF-IIS-0812194 and by the National Aeronautics and Space Administration (NASA) under the grant number NASA-AIST-08-0081.
}

Permission to make digital or hard copies of all or part of this work for personal or classroom use is granted without fee provided that copies are not made or distributed for profit or commercial advantage and that copies bear this notice and the full citation on the first page. To copy otherwise, to republish, to post on servers or to redistribute to lists, requires prior specific permission and/or a fee.

ACM GIS '10, November 2-5, 2010. San Jose, CA, USA

Copyright 2010 ACM 978-1-4503-0428-3/10/11 ...\$10.00. modifications of their spatial relationships over time, called developments. In spatial databases and GIS, spatio-temporal queries are particularly interesting when they ask for temporal changes in the spatial relationships between moving objects. An important class of spatial relationships are cardinal directions like north and southeast that characterize the relative directional position between spatial objects. Cardinal directions between two static objects have been extensively studied and have been frequently used as selection and join criteria in spatial queries. Transferred to a spatio-temporal context, the simultaneous location change of different moving objects can imply a change of their directional relationships. For example, a fishing boat that is southwest of a storm might be north of it some time later. We call this a cardinal direction development. Such a development between two moving objects describes a temporally ordered sequence of cardinal directions where each cardinal direction holds for a certain time interval during their movements. A development reflects the impact of time on the directional relationships between two moving objects, and usually proceeds continuously over time if the movements of the two objects are continuous.

It is an open, interesting, and challenging problem to capture the cardinal direction development between moving objects. Consider a spatio-temporal database containing information about weather conditions. Assuming a relation hurricane with attributes name of type integer and route of type mpoint (the moving point data type), the query whether a hurricane stayed all the time to the southeast of another hurricane can be formulated in an SQL-like style as follows:

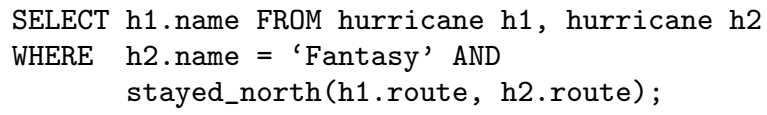

Queries like this can be particularly interesting to hurricane researchers to understand dynamic weather movement patterns. However, to answer this query with current approaches and systems, we would need to check the validity of the spatial directional predicate, e.g. southeast, at all time instances during the common life time of both hurricanes. This is not possible since the movements of the hurricanes are continuous. The fact that the traditional, static cardinal directions cannot describe continuous, time dependent relationships leads to the need for new modeling strategies.

From a modeling perspective, we define the development of cardinal directions over time as a sequence of temporally 
ordered and enduring cardinal directions. We have also developed algorithms for computing the development of cardinal directions, but due to the page limitation, our goal for this paper is restricted to the conceptual modeling stratergy.

Section 2 introduces the related work in the literature. In Section 3, we present the formal definition for cardinal direction developments. We draw some conclusions and discuss future work in Section 4.

\section{RELATED WORK}

Qualitative spatial relationships have a long tradition in GIS and spatial databases. They can be grouped into three categories: topological, directional and distance. The same classification holds for the relationships between moving objects. The distinction is that spatial relationships between moving objects can have temporal evolution, i.e., they may change over time. So far, the focus has been mainly on spatiotemporal topological relationships (like cross and enter) and spatio-temporal distance relationships (like moving towards, moving away from) and opposite_direction. Cardinal directions in a spatio-temporal context have been largely neglected in the literature. Static cardinal directions like north and northeast represent important qualitative spatial relationships that describe relative direction positions between static spatial objects. Many models follow a projection-based approach, where directional relationships are defined using projection lines orthogonal to the coordinate axes $[1,5]$. Some models apply a cone-based approach that defines directional relations by using angular zones $[2,6]$. Others like the Minimum Bounding Rectangle (MBR) model [4] make use of the minimum bounding rectangles of both operand objects and apply Allen's 13 interval relations to the rectangle projections on the $x$ - and $y$-axes respectively. However, all existing cardinal direction models only consider static directional relationships, and when transferred to a spatiotemporal context, none of the models is capable of modeling directional relationships that continuously change over time. In [3], an attempt has been made to model moving spatio-temporal relationships (mst-relation), which includes both topological relations and directional relations. During a time interval $I_{k}$, the mst-relation between two moving objects $A_{i}$ and $A_{j}$ is expressed as $A_{i}\left(\alpha, \beta, I_{k}\right) A_{j}$, where $\alpha$ is any topological relation among Equal, Inside, Contain, Cover, Covered By, Overlap, Touch and Disjoint and $\beta$ is one of the 12 directional relations, South, North, West, East, Northwest, Northeast, Southwest, Southeast, Left, Right, Below and Above. Both $A_{i} \alpha A_{j}$ and $A_{i} \beta A_{j}$ are true during the interval $I_{k}$. This model provides a way of describing the topological and directional relationships between two moving objects. However, it is not clear how the relationships are determined. There are currently no well established strategies for modeling cardinal directions between two moving objects; it is the main goal of this paper to bridge this gap.

\section{MODELING THE DEVELOPMENTS OF CARDINAL DIRECTIONS BE- TWEEN MOVING POINTS}

In Section 3.1, we first review the definitions for cardinal directions between static points without the consideration of time. Then, in Section 3.2, we model the temporal evolution of the cardinal directions between two moving points as a cardinal direction development.

\subsection{Cardinal Directions between Static Points}

The approach that is usually taken for defining cardinal directions between two static points in the Euclidean plane is to divide the plane into partitions using the two points. One popular partition method is the projection-based method that uses lines orthogonal to the $x$ - and $y$-coordinate axes to make partitions $[4,1]$. The point that is used to create the partitions is called the reference point, and the other point is called the target point. The directional relation between two points is then determined by the partition that the target object is in, with respect to the reference object. Let Points denote the set of static point objects, and let $p, q \in$ Points be two static point objects, where $p$ is the target point and $q$ is the reference point. A total of 9 mutually exclusive cardinal directions are possible between $p$ and $q$. Let $C D$ denote the set of 9 cardinal directions, then $C D=\{$ northwest $(N W)$, restrictednorth $(N)$, northeast $(N E)$, restrictedwest $(W)$, sameposition $(S P)$, restrictedeast $(E)$, southwest $(S W)$, restrictedsouth $(S)$, southeast $(S E)\}$. Further, let $X$ and $Y$ be functions that return the $x$ and $y$ coordinate of a point object respectively. The cardinal direction $\operatorname{dir}(p, q) \in C D$ between $p$ and $q$ is therefore defined as

$$
\operatorname{dir}(p, q)= \begin{cases}N W & \text { if } X(p)<X(q) \wedge Y(p)>Y(q) \\ N & \text { if } X(p)=X(q) \wedge Y(p)>Y(q) \\ N E & \text { if } X(p)>X(q) \wedge Y(p)>Y(q) \\ W & \text { if } X(p)<X(q) \wedge Y(p)=Y(q) \\ S P & \text { if } X(p)=X(q) \wedge Y(p)=Y(q) \\ E & \text { if } X(p)>X(q) \wedge Y(p)=Y(q) \\ S W & \text { if } X(p)<X(q) \wedge Y(p)<Y(q) \\ S & \text { if } X(p)=X(q) \wedge Y(p)<Y(q) \\ S E & \text { if } X(p)>X(q) \wedge Y(p)<Y(q)\end{cases}
$$

\subsection{The Development of Cardinal Direc- tions between Two Moving Points}

When two points change their locations over time, the directional relation between them becomes time related, and may or may not change. First, we consider the cardinal directions at time instances. Let time denote the temporal data type representing time and MPoints denote the spatio-temporal data type that represents moving points. For $A, B \in$ MPoints, let $A(t)$ and $B(t)$ denote the snapshots of $A$ and $B$ at a time instance $t \in$ time. If both $A$ and $B$ are defined at time $t$, then $A(t), B(t) \in$ Points. The cardinal direction between $A$ and $B$ at $t$ is therefore $\operatorname{dir}(A(t), B(t)) \in C D$. For example, in Figure 1a, at time $t_{1}$ when $A$ and $B$ locate at $A\left(t_{1}\right)$ and $B\left(t_{1}\right)$, the cardinal direction between $A$ and $B$ at time instance $t_{1}$ is $\operatorname{dir}\left(A\left(t_{1}\right)\right.$, $\left.B\left(t_{1}\right)\right)=N W$. At the time instance $t_{2}$ when $A$ and $B$ move to $A\left(t_{2}\right)$ and $B\left(t_{2}\right)$, the cardinal direction between them becomes $\operatorname{dir}\left(A\left(t_{2}\right), B\left(t_{2}\right)\right)=S E$. We propose our solution to determine what happened in between and to answer the question whether there exists a time instance $t\left(t_{1}<t<t_{2}\right)$ such that $\operatorname{dir}(A(t), B(t))=W$ in the following sections. This 


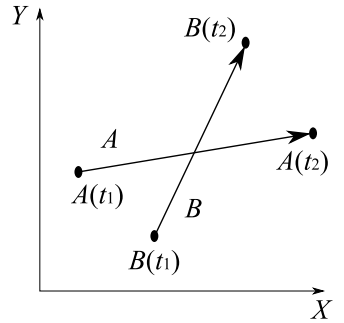

(a)

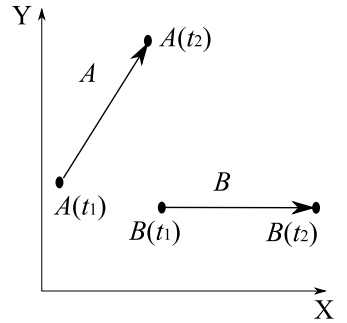

(b)
Figure 1. An example of two moving points with changed (a) and unchanged (b) cardinal directions over time

scenario shows that within a common time interval, we may get different cardinal directions at different time instances. However, the change of time does not necessarily imply the change of cardinal directions between two moving points. In Figure 1b two moving points $A$ and $B$ start at time $t_{1}$ from the locations $A\left(t_{1}\right)$ and $B\left(t_{1}\right)$ respectively. At time $t_{2}, A$ reaches the location $A\left(t_{2}\right)$ and $B$ reaches the location $B\left(t_{2}\right)$. One observation that we can obtain is that although the positions of $A$ and $B$ have changed, the cardinal direction between $A$ and $B$ does not change. In this case, $A$ is always to the northwest of $B$ between $t_{1}$ and $t_{2}$. In other words, the cardinal direction between two moving points holds for a certain period of time before it changes. Thus, we define a predicate holds that returns true if a cardinal direction holds during a time interval for two moving points $A$ and $B$.

Definition 1. Given two moving points $A, B \in M$ Point, a time interval $I=\left[t_{b}, t_{e}\right]$ with $t_{b}, t_{e} \in$ time and $t_{b} \leq t_{e}$, and the basic cardinal direction set CD. Assume both $A$ and $B$ are defined on $I$. Let holds be the predicate that returns true if $d \in C D$ holds over the time interval $I$ for $A$ and $B$. We define this function as

$$
\operatorname{holds}(A, B, I, d)= \begin{cases}\text { true } & \text { if } \forall t \in I: \\ & \operatorname{dir}(A(t), B(t))=d \\ \text { false } & \text { otherwise }\end{cases}
$$

For a given time interval $I$, we make the following observations: (i) if there exists a cardinal direction $d \in C D$ such that $h o l d s(A, B, I, d)=$ true, then we say $A$ and $B$ have a unique cardinal direction on the time interval $I$; (ii) if both $A$ and $B$ are defined on $I$, and the predicate holds returns false for all 9 basic cardinal directions in $C D$, then we say that $A$ and $B$ have a developing cardinal direction relationship over $I$; (iii) if neither $A$ nor $B$ is defined on $I$, we say the cardinal direction between $A$ and $B$ is not defined on $I$. Therefore, we can only determine cardinal directions between $A$ and $B$ during intervals on which they are defined. For an interval where there is no unique basic cardinal direction that holds over the entire period, we split it into several sub-intervals such that on each sub-interval only a unique cardinal direction holds.

Further, if we regard different cardinal directions that hold over different sub-intervals as cardinal direction states, the development of the cardinal directions refers to a sequence of transitions between these states. For example, $A$ moving from $N W$ to $W$ to $S W$ of $B$ is a development of cardinal directions between two moving points $A$ and $B$. However, not all transitions are possible between any

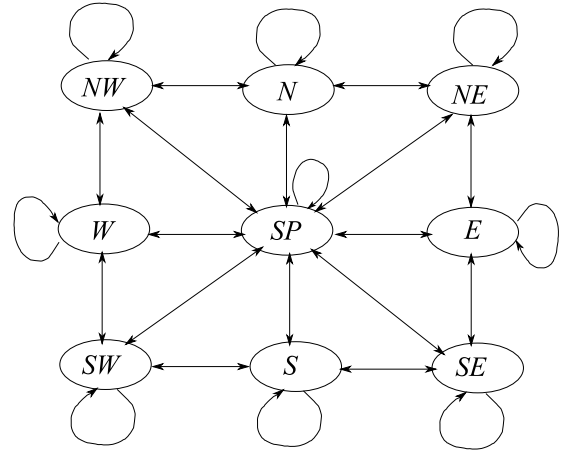

Figure 2. The state transition diagram of all cardinal directions

two states. Figure 2 shows all possible transitions between different states. For example, if the cardinal direction between two moving points $A$ and $B$ has been $N W$ so far, then if time changes, the cardinal direction might stay the same as $N W$, or change to either $N, W$, or $S P$. It is not possible that $A$ moves directly to the south $(S)$ of $B$ without crossing any other directions. This state transition diagram implies that only developments that involve valid transitions are possible between two moving points, e.g, $A$ moves from $N W$ to $W$ to $S W$ of $B$. Developments that involve invalid transitions like $A$ moving from $N W$ to $S$ of $B$ are not possible and thus not allowed. Let the predicate isValidTrans : $C D \times C D \rightarrow$ bool take two cardinal directions as input, and yield true if the transition between them is valid. Then, for example, isValidTrans $(N W, W)=$ true while isValidTrans $(N W, S)=$ false. Now we can define the development of the cardinal directions between two moving points $A$ and $B$ on any given time interval $I$ on which $A$ and $B$ are both defined.

Definition 2. Given two moving points $A, B \in$ MPoints and a time interval $I$ on which both $A$ and $B$ are defined. Assume the ordering of any two intervals $I_{1}=\left[t_{b 1}, t_{e 1}\right]$ and $I_{2}=\left[t_{b 2}, t_{e 2}\right]$ is defined as $t_{e 1} \leq t_{b 2} \Leftrightarrow I_{1} \leq I_{2}$. Let the symbol $\triangleright$ represent the transition from one cardinal direction state to another. Then the development of cardinal directions between $A$ and $B$ on interval $I$, denoted as $\operatorname{dev}(A, B, I)$ can be defined as:

$$
\operatorname{dev}(A, B, I)=d_{1} \triangleright d_{2} \triangleright \ldots \triangleright d_{n}
$$

if the following conditions hold:

(i) $n \in \mathbb{N}$

(ii) $\forall 1 \leq i \leq n: d_{i} \in C D$

(iii) $\forall 1 \leq i \leq n-1: d_{i} \neq d_{i+1}$

$$
\text { isValidTrans }\left(d_{i}, d_{i+1}\right)=\text { true }
$$

(iv) $\exists I_{1}, I_{2}, \ldots, I_{n}$ :

$$
\begin{aligned}
& \text { (a) } \forall 1 \leq i \leq n: I_{i} \text { is a time interval, } \\
& \text { holds }\left(A, B, I_{i}, d_{i}\right)=\text { true } \\
& \text { (b) } \forall 1 \leq i \leq n-1: I_{i} \leq I_{i+1} \\
& \text { (c) } \bigcup_{i=1}^{n} I_{i}=I
\end{aligned}
$$

In Definition 2, we split the given time interval into a sequence of non-overlapping sub-intervals. The development dev represents the transition of cardinal directions over these 


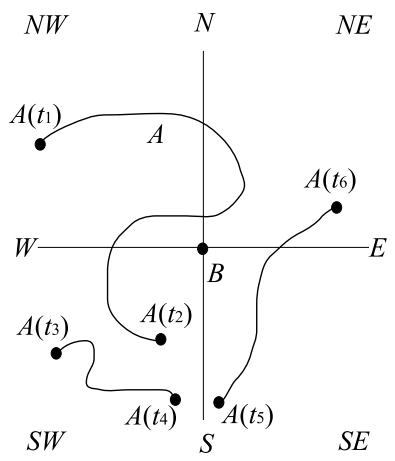

Figure 3. An example of a cardinal direction development

sub-intervals. Condition (iv)(a) ensures that a unique cardinal direction between $A$ and $B$ holds on each sub-interval, and condition (iv)(c) ensures that all sub-intervals together form a full decomposition of the given interval $I$. Further, according to condition (iii), only valid transitions are allowed between two cardinal directions that hold on adjacent sub-intervals. An example of such a development can be derived from Figure 3, where $A$ moves from location $a_{1}$ to location $a_{2}$ and $B$ does not move during the time interval $I=\left[t_{1}, t_{2}\right]$. The development of cardinal directions between $A$ and $B$ during $I$ is therefore $\operatorname{dev}(A, B, I)=$ $N W \triangleright N \triangleright N E \triangleright N \triangleright N W \triangleright W \triangleright S W$. It describes that from time $t_{1}$ to time $t_{2}, A$ starts in $N W$ of $B$, crosses $N$ and reaches $N E$ of $B$, then it turns around and crosses $N$ again, and returns to $N W$ of $B$. Finally, $A$ crosses $W$ of $B$ and ends up in the $S W$ of $B$.

Now we are ready to define cardinal direction developments between two moving points during their entire life time. The idea is to first find out their common life time intervals, on which both $A$ and $B$ are defined. Then we apply the dev function to determine the development of cardinal directions between $A$ and $B$ during each common life time interval. Finally, we compose the cardinal direction developments on different common life time intervals and define it as the development of cardinal directions between the two moving points $A$ and $B$. We first find out the common life time intervals for two moving points.

Definition 3. Given two moving points $A, B \in$ MPoints, let $L T_{A}=\left\langle I_{1}^{A}, I_{2}^{A}, \ldots, I_{m}^{A}\right\rangle, L T_{B}=\left\langle I_{1}^{B}, I_{2}^{B}, \ldots, I_{n}^{B}\right\rangle$ be two life time interval sequences of $A$ and $B$ respectively such that $I_{i}^{A}<I_{i+1}^{A}$ and $I_{j}^{B}<I_{j+1}^{B}$ with $1 \leq i \leq m, 1 \leq j \leq n$. We now define the common life time $C L T$ for $A$ and $B$ as

$$
C L T(A, B)=\left\langle I_{1}, I_{2}, \ldots, I_{l}\right\rangle
$$

if the following conditions hold:

$$
\begin{aligned}
& \text { (i) } l<n+m \\
& \text { (ii) } \forall 1 \leq i \leq l: I_{i} \in \bigcup_{j=1}^{m} \bigcup_{k=1}^{n}\left(I_{j}^{A} \cap I_{k}^{B}\right) \\
& \text { (iii) } \forall 1 \leq i<l: I_{i}<I_{i+1}
\end{aligned}
$$

Definition 3 defines the common life time of $A$ and $B$ as a list of intervals on which both $A$ and $B$ are defined. Directional relationships between $A$ and $B$ only exist during their common life time. At any time instance that is not within their common life time, the cardinal direction between $A$ and $B$ is not defined. For example, in Figure 3, since $B$ is assumed to exist all the time, the common life time of $A$ and $B$ is $C L T(A, B)=\left\langle\left[t_{1}, t_{2}\right],\left[t_{3}, t_{4}\right],\left[t_{5}, t_{6}\right]\right\rangle$. During the time interval $\left(t_{2}, t_{3}\right)$, the moving point $A$ is not defined; thus it is not part of the common life time of $A$ and $B$. Finally, the development of cardinal directions between $A$ and $B$ can be defined as

Definition 4. Given two moving points $A, B \in$ MPoints and $C L T(A, B)=\left\langle I_{1}, I_{2}, \ldots, I_{l}\right\rangle$. Let the symbol $\perp$ represent the meaning of undefined direction. Then the development of cardinal directions between $A$ and $B$, denoted as $D E V(A, B)$ can be defined as:

$$
\begin{aligned}
\operatorname{DEV}(A, B)= & \operatorname{dev}\left(A, B, I_{1}\right) \triangleright \perp \triangleright \operatorname{dev}\left(A, B, I_{2}\right) \\
& \triangleright \perp \triangleright \ldots \triangleright \operatorname{dev}\left(A, B, I_{n}\right)
\end{aligned}
$$

Definition 4 generalizes the development of cardinal directions between two moving points from a given interval to their entire life time. The cardinal direction development between $A$ and $B$ in Figure 3 is therefore $\operatorname{DEV}(A, B)=$ $N W \triangleright N \triangleright N E \triangleright N \triangleright N W \triangleright W \triangleright S W \triangleright \perp \triangleright S W \triangleright \perp \triangleright S E \triangleright E \triangleright N E$.

\section{CONCLUSIONS AND FUTURE WORK}

In this paper, we have laid the foundation of a novel concept, called cardinal direction development, for determining the evolution of cardinal directions between moving points. We have proposed a formal definition that clarifies the concept from an abstract point of view. We have also developed a three-phase solution for computing the cardinal directions between two moving points from an algorithmic perspective. However, due to the space limitation, it is not in the scope of this paper.

In the future, we plan to extend our concept to more complex moving objects like moving regions and moving lines. We will also take into account that moving objects can consist of several components.

\section{References}

[1] A. Frank. Qualitative Spatial Reasoning: Cardinal Directions As an Example. International Journal of Geographical Information Science, 10(3):269-290, 1996.

[2] R. Haar. Computational Models of Spatial Relations. Technical Report: TR-478, (MSC-72-03610), 1976.

[3] J. Z. Li, M. T. Ozsu, M. Tamer, D. Szafron, and S. G. Ddi. Modeling of Moving Objects in a Video Database. In IEEE International Conference on Multimedia Computing and Systems, pages 336-343, 1997.

[4] D. Papadias, Y. Theodoridis, and T. Sellis. The Retrieval of Direction Relations Using R-trees. In Int. Conf. on Database and Expert Systems Applications (DEXA), pages 173-182, 1994.

[5] S. Skiadopoulos and M. Koubarakis. Composing Cardinal Direction Relations. Artificial Intelligence, 152:143171, 2004.

[6] S. Skiadopoulos, N. Sarkas, T. Sellis, and M. Koubarakis. A Family of Directional Relation Models for Extended Objects. IEEE Trans. on Knowledge and Data Engineering (TKDE), 19, 2007. 